\title{
3D Imaging of Porous Media using Laser Scanning Confocal Microscopy with Application to Microscale Transport Processes
}

\author{
J. T. Fredrich \\ Sandia National Laboratories, Albuquerque, New Mexico $\quad$ ECIVED \\ FEB 181999 \\ Q S 1 \\ Camera-ready Copy for \\ Physics and Chemistry of the Earth \\ Manuscript No. SE39.2-001 \\ Solicited Manuscript (extended) \\ Offset requests to: \\ J.T. Fredrich \\ Geomechanics Department 6117 \\ Sandia National Laboratories \\ Albuquerque, NM 87185-0751 \\ USA
}

January 27, 1999 


\section{DISCLAIMER}

This report was prepared as an account of work sponsored by an agency of the United States Government. Neither the United States Government nor any agency thereof, nor any of their employees, make any warranty, express or implied, or assumes any legal liability or responsibility for the accuracy, completeness, or usefulness of any information, apparatus, product, or process disclosed, or represents that its use would not infringe privately owned rights. Reference herein to any specific commercial product, process, or service by trade name, trademark, manufacturer, or otherwise does not necessarily constitute or imply its endorsement, recommendation, or favoring by the United States Government or any agency thereof. The views and opinions of authors expressed herein do not necessarily state or reflect those of the United States Government or any agency thereof. 


\section{DISCLAIMER}

Portions of this document may be illegible in electronic image products. Images are produced from the best available original document. 


\title{
$3 \mathrm{D}$ imaging of porous media using laser scanning confocal microscopy with application to microscale transport processes
}

\author{
J. T. Fredrich \\ Sandia National Laboratories, Albuquerque, New Mexico
}

Received 1 November 1998 - Accepted 7 December 1998

\begin{abstract}
We present advances in the application of laser scanning confocal microscopy (LSCM) to image, reconstruct, and characterize statistically the microgeometry of porous geologic and engineering materials. We discuss technical and practical aspects of this imaging technique, including both its advantages and limitations. Confocal imaging can be used to optically section a material, with sub-micron resolution possible in the lateral and axial planes. The resultant volumetric image data, consisting of fluorescence intensities for typically $\sim 50$ million voxels in $\mathrm{XYZ}$ space, can be used to reconstruct the three-dimensional structure of the two-phase medium. We present several examples of this application, including studying pore geometry in sandstone, characterizing brittle failure processes in low-porosity rock deformed under triaxial loading conditions in the laboratory, and analyzing the microstructure of porous ceramic insulations. We then describe approaches to extract statistical microgeometric descriptions from volumetric image data, and present results derived from confocal volumetric data sets. Finally, we develop the use of confocal image data to automatically generate a three-dimensional mesh for numerical pore-scale flow simulations.
\end{abstract}

\section{Introduction}

Natural as well as many engineering materials exhibit complex pore structures which greatly influence their bulk physical and mechanical properties, including elastic moduli, compressibility, seismic velocity, permeability, electrical conductivity, thermal conductivity, poroelastic parameters, strength, and failure behavior. The extent to

Correspondence to: J.T. Fredrich, Mail Stop 0751, Sandia National Laboratories, Albuquerque, NM 87185-0751 (email: fredrich@sandia.gov) which the macroscopic properties of an arbitrarily complex porous medium can be predicted using a microscale modeling approach follows directly from the sophistication of the model, which in turn relies on the completeness of the microgeometric description available as input.

The transport properties in particular depend critically on the geometry of the void space (e.g., Adler, 1992; Dullien, 1992; Sahimi, 1995). The size, shape, connectivity, and tortuosity of the pore space can in fact affect the permeability of a porous medium more strongly than the total void fraction available to transmit that flow. As an example, consider a typical quartz sandstone such as Berea with a bulk porosity of $\sim 20-25 \%$ and a permeability of $\sim 200-800$ millidarcy. Contrast that with a sedimentary diatomaceous rock with more than twice the porosity $(\sim 60 \%)$, but a permeability that is lower by well over two orders of magnitude ( 0.1-1 millidarcy). This dramatic inversion of the usual trend between porosity and permeability is a direct consequence of differences in the geometry of the pore space for the two rocks.

A fundamental understanding of flow processes in complex porous media requires both statistical characterization of the geometry of the porous microstructure, as well as study of the geometry of the flow itself. The former aspect can in principle be addressed experimentally; however, the geometry of the flow through a complex porous medium is difficult to ascertain in the laboratory, but amenable to numerical investigation. Using idealized network models, David (1993) demonstrated the complexity of hydraulic flow paths in heterogeneous porous media, and that the critical paths for hydraulic flow are not necessarily identical to those for electric current.

A variety of geoscience and engineering applications stand to benefit significantly from an improved understanding of transport processes in geometrically complex porous media. Geoscience applications include understanding the displacement of oil from the pore space during waterflooding or enhanced oil recovery processes, 
and predicting the transport of contaminants in an underground aquifer. Understanding the physics of microscale flow processes is likewise central to many chemical, materials, and thermal engineering applications, including porous bed chemical reactors, powder compaction and sintering, gas flow in thermal protection systems, heat dissipation in microelectronic components, and fluid and thermal flows in MicroElectroMechanical Systems (MEMS).

We believe that an improved understanding requires simultaneous progress on two fronts: 1) new experimental techniques to characterize the three-dimensional microgeometry of complex porous media; and 2) efficient and accurate numerical methods to model and simulate mass and heat transfer processes in complex porous media. This paper summarizes our progress in the first area, and $O^{\prime}$ Connor and Fredrich (this volume) describe our recent work in the latter.

\section{Microstructural characterization of porous media}

The simplest geometric attribute of a porous material is its porosity, or void fraction, $\phi$. This property can be measured on bulk samples to high accuracy using gas porosimetry techniques based on Boyle's Law, although less precise imbibition (saturation) techniques are also commonly applied. Specific surface area $S_{A}$, defined as the grain-void interface area normalized by the total volume, is the next most elementary property characterizing a porous medium. $S_{A}$ as well can be readily measured on a bulk sample using gas adsorption techniques such as the BET method (Brunauer et al, 1938). Limited additional geometric information can be obtained from bulk samples using mercury porosimetry (e.g., Van Brakel et al., 1981), smallangle scattering (Wong et al., 1986; Hall et al., 1986), adsorption-desorption or other extensions of the basic gas adsorption technique (e.g., Avnir et al., 1984), or nuclear magnetic resonance (Cohen and Mendelson, 1982; Mendleson, 1982). For geologic materials, mercury porosimetry is probably the most commonly applied indirect technique; information concerning "effective" pore sizes can be derived from the capillary pressure record. However, the geometric complexity of the pore space (i.e. its interconnectivity, geometric irregularity, etc.) leads to ambiguities in the physical interpretation of mercury capillary data, and the other indirect methods likewise require certain geometric assumptions that complicate their interpretation.

Imaging methods offer the possibility to characterize unambiguously, and completely, the microstructure of a porous material. Conventional techniques include reflected or transmitted light microscopy (LM) and scanning electron microscopy (SEM) of planar sections. Quantitative stereological methods (Underwood, 1970; Russ, 1986) can be applied to determine geometric parameters such as porosity and specific surface area directly from measurements made on planar sections (e.g., Wong, 1985). Similarly, stereological measurements of chord length can
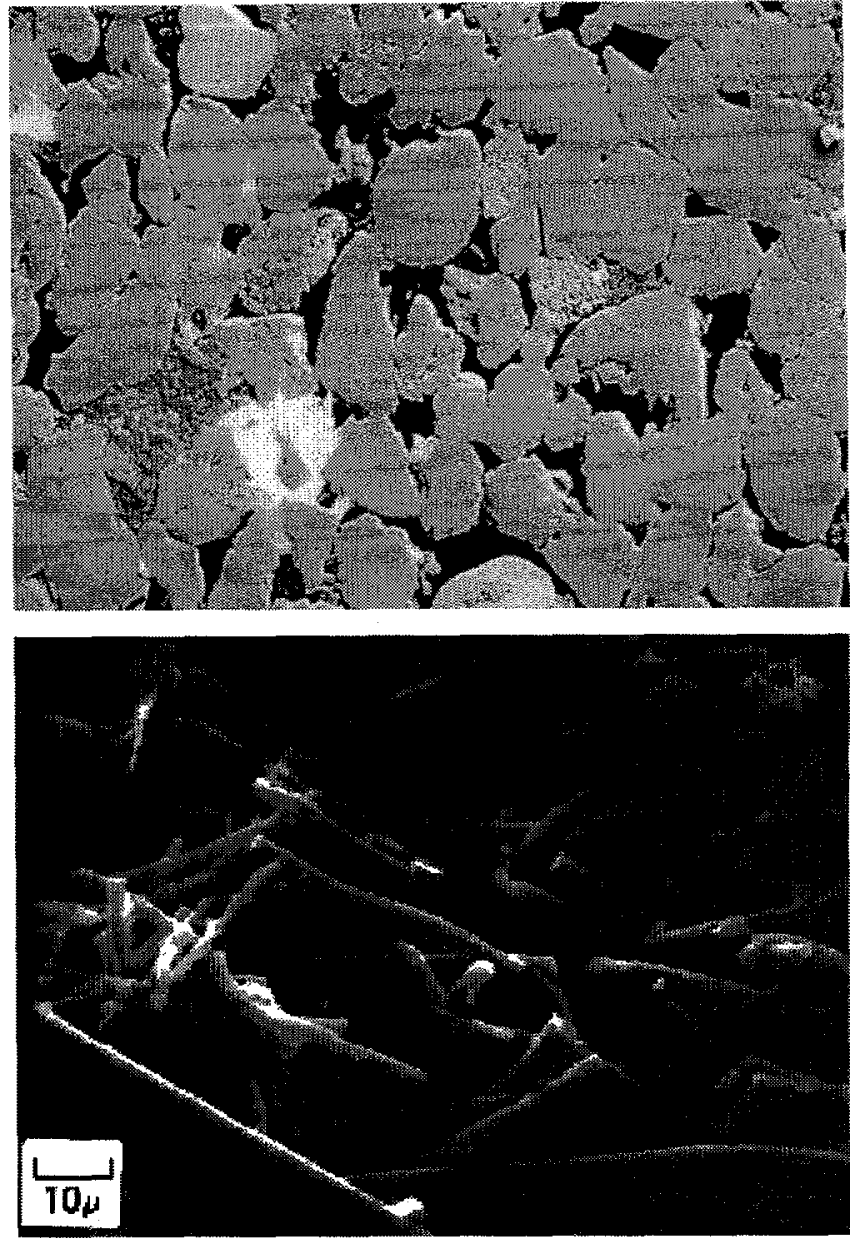

Fig. 1. Scanning electron micrographs of (top) Berea sandstone (backscattered imaging mode), and (bottom) a porous rigid fibrous refractory insulation. The sandstone has a grain size of $\sim 150$ micron, and the pore space has been impregnated with epoxy (black). There is no continuous path through the pore phase in the imaging plane. The insulation is composed of ceramic fibers that occupy less than $\sim 20 \%$ of the bulk volume, and that have a diameter of several microns. The image reveals the complex geometry of both the solid and pore phases, but it is unfeasible to extract a quantitative description of the three-dimensional microstructure. (Bottom image courtesy of NASA Ames Research Center, Thermal Protection Materials and Systems Branch)

be used to infer pore and crack size distributions (Krohn, 1988; Wong et al., 1989; Fredrich et al., 1993). Alternatively, both porosity and specific surface area can be calculated from the 2-point probability function (defined later) that can be measured from micrographs of polished sections that have been processed to yield binary data (Berryman, 1985; Berryman and Blair, 1986). Methods have also been devised to characterize the fractal properties of the pore space using measurements made on images of polished sections (Thompson, 1991), as well as from images of rough fracture surfaces (Krohn and Thompson, 1986).

An important handicap of the traditional imaging approaches (Figure 1), however, is that one is restricted to examining a two-dimensional (2D) representation of a three-dimensional (3D) object, which greatly complicates, if not precludes, a determination of the true threedimensional structure. As was pointed out by Bernabe 
(1991), certain pore types (such as tubular pores) are difficult to identify in $2 \mathrm{D}$ images. Likewise, it is impossible to determine the connectivity of a pore network from a $2 \mathrm{D}$ image. A quick examination of a 2D micrograph (Figure 1, Top) from a typical sandstone shows that the pore phase is discontinuous in the two-dimensional plane of the image; however, we know with certainty by the presence of the epoxy that the pore phase is in actuality connected in three dimensions.

Several innovative techniques have been developed by researchers to circumvent this limitation. Pore casts have been formed by impregnating a rock with an acid-resistant epoxy and then dissolving away the mineral matrix with acid (Pittman and Duschatko, 1970). The pore casts can then be imaged using SEM, and while they provide useful insight (e.g., Bourbie and Zinszner, 1985; Myer et al., 1992; Zinszner et al., 1997), the stereoscopic images are difficult to quantify. The same problem arises during a SEM study of the rough or irregular surface of a porous material itself (Figure 1, Bottom). Difficulties may also be encountered in impregnating and preserving the delicate pore structure of low porosity samples (Lin et al., 1986).

Koplik et al. (1984) made sequential optical micrographs of a planar section after incrementally removing as little as $1 \mu \mathrm{m}$ of material by polishing. Lin et al. (1986) coupled such serial sectioning with image processing techniques to generate 3D images of porosity in a tight gas sand using conventional optical microscopy. Although effective, this technique is time consuming and tedious.

In the past ten or so years several new approaches have emerged for imaging the 3D structure of porous media. Xray computed tomography (CT), originally developed by the medical community, can be used to measure porosity and to image porosity distribution in bulk porous samples (e.g., Wellington and Vinegar, 1987). However, quantitative measurements are complicated greatly by the need for beam hardening corrections that arise due to the polychromatic nature of X-ray sources, and that are particularly challenging for heterogeneous materials (Brown et al., 1993; Fredrich et al., 1994). Moreover, the typical resolution of $0.1-1 \mathrm{~mm}$ is not sufficient for detailed characterization of most porous media of interest in the geo- and materials- sciences. Brown et al. (1993) described a gamma ray $\mathrm{CT}$ system that relied on a monochromatic source, thereby obviating the need for complicated beam hardening relationships. However, the system resolution of $1 \mathrm{~mm}$ again indicates that while conventional $X$-ray and gamma ray $\mathrm{CT}$ are useful techniques for studying transport in fractured rock, they are not generally effective for characterizing porous media.

Nuclear magnetic resonance imaging (MRI), also developed primarily for medical uses, has seen increasing application for characterizing the structure of porous materials (Baldwin et al., 1996; Doughty and Tomutsa, 1997). However, while significantly higher resolution than $\mathrm{X}$-ray CT, the current maximum resolution of $\sim 25 \mu \mathrm{m}$ is not sufficient to capture the narrow pore throats characteristic of most geologic media. For example, even in porous sandstone such as Berea or Fontainebleau, pore throats as narrow as a few microns are statistically significant (Doyen, 1988; Fredrich et al., 1993). Besides being thought to control bulk transport properties such as permeability (e.g., Yale, 1985; Doyen, 1988; Bernabe, 1991), the compliant pore throats also impact critically the evolution of permeability with changes in pore or confining pressure (e.g., Walsh and Brace, 1984), an application of considerable importance in the geosciences.

3D imaging of porous media has recently been accomplished using synchrotron computed microtomography, with data at a resolution of $10 \mu \mathrm{m}$ reported by Spanne et al. (1994), and data at a resolution of $7.5 \mu \mathrm{m}$ reported by both Auzerais et al. (1996) and Coker et al. (1996). This resolution is only slightly greater than the resolution of $1-3 \mu \mathrm{m}$ that is probably optimal for characterizing rocks such as sandstone. The tomographic reconstruction process favors the use of cylindrical samples and to obtain the quoted resolutions, the above workers used epoxy-impregnated samples ranging from 3 to $3.5 \mathrm{~mm}$ in diameter. While increased resolution is desirable and potentially feasible (e.g., Coker et al., 1996), probably the most important factor limiting routine application of this technique is facility access and the availability of beam-line time.

Laser scanning confocal microscopy (LSCM) is a relatively new optical imaging technique that has been exploited extensively in the biological community over the past decade (Pawley, 1990). Although the theoretical conception dates back over thirty years to Marvin Minski, routine application has only been made possible by recent advances in optical and electronic technology (Inoue, 1990). Three-dimensional imaging is possible because LSCM can be used to nondestructively "slice" thin optical sections with high axial resolution and precise registration.

\section{Principles of laser scanning confocal microscopy}

The unique optical sectioning capability of LSCM results from fundamental differences in image formation as compared to conventional light microscopy (LM). The essential feature of LSCM (Figure 2) is that both illumination and detection are confined to a single location on the specimen at any one time. This is achieved by the use of an hourglass-shaped beam to illuminate only a small volume at any single time, and by the insertion of a pinhole, or confocal, aperture in the returning optical path that blocks light emanating from planes above and below the focal plane. Because the confocal aperture admits light from only a specific plane of interest, thin optical sections can be resolved by varying the depth of the focal plane in the sample. (Conversely, light from both above and below the focal plane is admitted for image formation in conventional LM; this precludes the possibility for depth discrimination and also results in significant image degradation.) Because each point in the focal (image) plane is examined individually in LSCM (in the absence of light scattered from neighboring points), the lateral (in-plane, or $x-y$ ) resolution is greatly enhanced. (In conventional LM, an 


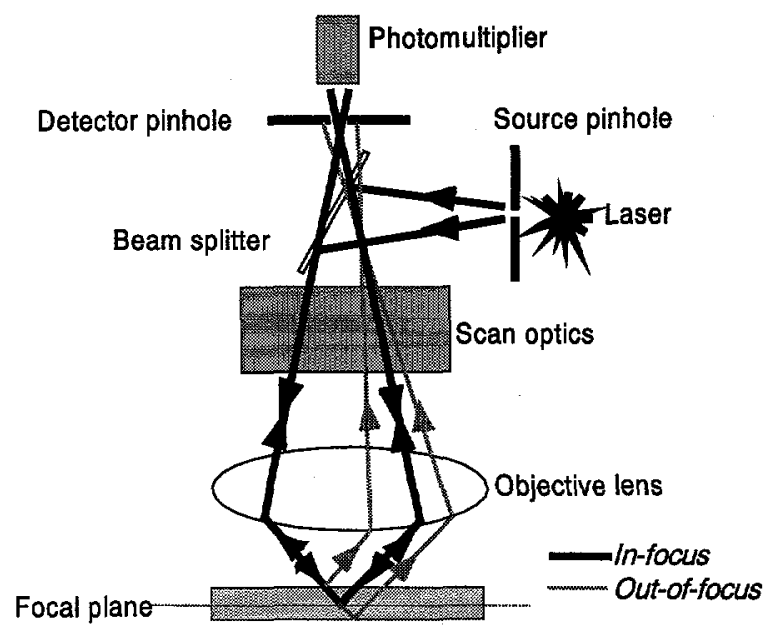

Fig. 2. Schematic illustrating the principle of laser scanning confocal microscopy.

object is viewed under uniform illumination and the point to point resolution is degraded by scattering). In LSCM, imaging of a plane (or volume) is accomplished by scanning sequentially in a point to point manner. The scanning of the laser in the horizontal $(x-y)$ plane is typically accomplished with a set of mirrors, whereas the vertical position of the stage (and therefore focal plane) is controlled with a precision stepper motor. Finally, the use of a laser light source in confocal systems further enhances resolution because lasers offer a high degree of monochromaticity, small divergence, high brightness, high degree of spatial and temporal coherence and plane polarized emission (Gratton and vandeVen, 1990).

The resultant lateral resolution $R$ is considerably improved over conventional LM, and has been demonstrated (Brakenhoff et al., 1979, 1989; Gard, 1993) to essentially reach the theoretical limit set by diffraction as defined by the Rayleigh criterion:

$$
R=0.61 \lambda / N A
$$

where $\lambda$ is the wavelength and $N A$ the numerical aperture of the objective.

The numerical aperture also determines the "thickness" of the optical section, with higher NA yielding "thinner" optical sections. In reality, the optical section does not have a discrete thickness, and is instead typically defined as the distance $z_{1 / 2}$ between the focus positions at which the collected intensity decays to one-half the peak value. As discussed above, the function of the confocal aperture is to exclude light emitted from above and below the focus plane, and the opening of the confocal aperture thus influences directly the vertical resolution of the optical section. The axial resolution is always less than the lateral resolution, and in practice (Brakenhoff et al., 1979; Gard, 1993) the maximum axial resolution (i.e., smallest $z_{1 / 2}$ or narrowest optical section) achieved in confocal systems varies from $\sim 3-10$ times the theoretical lateral resolution.
Table 1. Lateral resolution $R$ (Eq. 1$)$ and optical section thickness at $\lambda=514 \mathbf{n m}$ for various settings of the confocal aperture

\begin{tabular}{|c|c|c|c|c|c|c|}
\hline \multicolumn{2}{|c|}{ Objective } & \multirow[t]{2}{*}{$R(\mu \mathrm{m})$} & \multicolumn{4}{|c|}{ Section thickness $(\mu \mathrm{m})$} \\
\hline$M$ & $N A$ & & Open & $1 / 3$ & $2 / 3$ & Closed \\
\hline$\times 10$ & 0.45 & 0.71 & 38 & 25 & 13 & 7.0 \\
\hline$\times 20$ & 0.75 & 0.42 & 14 & 10 & 6.7 & 5.0 \\
\hline$\times 40$ & 1.0 & 0.31 & 6.1 & 4.2 & 2.6 & 1.4 \\
\hline$\times 60$ & 1.4 & 0.22 & 3.7 & 2.0 & 1.0 & 0.7 \\
\hline
\end{tabular}

With an objective of sufficiently high $N A$, sub-micron resolution in both the lateral and axial planes can be achieved (Wilson, 1989). Table 1 reproduces the optical section thickness experimentally measured by Gard (1993) on a confocal imaging system manufactured by Bio-Rad (MRC-600) and fitted to a Nikon Optiphot for various openings of the confocal aperture at $\lambda=514 \mathrm{~nm}$. In the MRC-600, the "closed" position corresponds to a minimum physical opening of $0.6 \mathrm{~mm}$, whereas the "open" setting corresponds to the maximum opening of $8 \mathrm{~mm}$.

\section{Technical and practical considerations}

Fredrich et al. (1993, 1995) and Montoto et al. (1995) independently described a technique for applying LSCM to image the void space of porous media. Here we provide additional technical details and discuss practical aspects important to consider for imaging porous materials.

Our technique consists of saturating the pore space of a bulk sample with a very low viscosity, slow-curing epoxy that is doped with a suitable fluorochrome. The wetting characteristics of the sample are generally improved if the sample is first flushed with a solvent such as acetone. Following vacuum drying of the sample, either vacuum impregnation or pressure impregnation can be used to force the epoxy into the void space.

We are partial to a four-component epoxy attributed to Spurr (1969) that has a viscosity of $\sim 60 \mathrm{cps}$ and an extended pot-life that approaches 24 hours (available from Ted Pella, Inc., Redding CA). Thorough mixing of the four components and dissolution of the fluorochrome (added as a powder) may take up to an hour, and air is typically incorporated during the process. The extended pot-life thus allows sufficient time both for complete degassing of the epoxy under vacuum prior to sample impregnation, and for the epoxy to penetrate completely into the void space during vacuum- or pressure- impregnation of the sample. Typically the sample is left overnight before curing at $60^{\circ} \mathrm{C}$. Finally, the sample is slabbed using a diamond wafering saw, mounted on a glass slide, and a polished planar surface is prepared using standard abrasive polishing techniques, typically to a final finish with $0.05 \mu \mathrm{m}$ alumina. The thickness is irrelevant. We have verified by direct observation that this procedure is sufficient to ensure epoxy penetration into cracks and pores at the resolution limit of the microscope $(200 \mathrm{~nm})$ for one-inch diameter cores of geologic samples ranging from sandstone to granite.

The choice of fluorochrome is significant (Wilson, 1990), and the absorption peak of the fluorochrome should be well 
matched to the imaging wavelength. Confocal systems most commonly come equipped with either an argon ion laser with lines at $488 \mathrm{~nm}$ and $514 \mathrm{~nm}$, or a krypton-argon mixed gas laser with lines at $488 \mathrm{~nm}, 568 \mathrm{~nm}$, and $647 \mathrm{~nm}$. In our work, we have used Rhodamine B (available from Polysciences Inc., Warrington PA), a popular fluorochrome that dissolves well in epoxy, usually at a concentration of 1:200 (rhodamine: total epoxy components) by mass. Rhodamine B is relatively resistant to photobleaching, and although it can be excited with the $514 \mathrm{~nm}$ line of an argon ion laser, it is excited more effectively with the $568 \mathrm{~nm}$ line of a krypton-argon mixed gas laser. The wider separation of lines possible with the krypton-argon mixed gas laser also facilitates dual excitation imaging. For example, one might impregnate the pore space with two different epoxies or resins that have been doped with different fluorochromes that are then both excited and distinguished individually using the $488 \mathrm{~nm}$ and $568 \mathrm{~nm}$ lines simultaneously.

During image collection, the fluorescence intensity as the laser is scanned point-to-point across the field of view is recorded. We find that data acquired at 8-bit resolution (0255 gray levels) is sufficient for our interests. Besides the obvious influence of fluorochrome concentration, there are three primary factors affecting the gray level, or brightness, of an image: the opening of the confocal aperture, the laser intensity, and the gain of the photomultiplier. The user must balance each of these simultaneously to arrive at an optimal solution. For example, a larger confocal aperture yields a brighter image, because a thicker optical section is being imaged. Increasing the laser intensity likewise increases image brightness; however, fluorochromes generally saturate at low laser intensity, and a high laser intensity also increases the risk of photobleaching (fading) of the fluorochrome. Finally, image brightness is improved by increasing the gain of the photomultiplier, but this also results in increased noise.

For the applications considered here there are two additional considerations. First, a major interest is to exploit the capability for optical sectioning, and clearly the laser is attenuated with depth due to both absorption and scattering by the material lying above the focal plane, so that greater laser intensity leads to greater penetration depth. Second, we are generally interested in minimizing the optical section thickness (Table 1) so that it is comparable to the lateral resolution. (As we discuss later on, subsequent image processing is facilitated if the data are collected to yield cubic voxels, a voxel being a three-dimensional volume element, i.e., the volumetric equivalent to the twodimensional pixel, or picture element.)

For the fluorochrome concentrations used in our work, filtering the laser to only $10 \%$ transmission results in observable photobleaching (using a krypton-argon mixed gas laser with about $5 \mathrm{~mW}$ emitted power on the $568 \mathrm{~nm}$ line). However, $3 \%$ transmission results in no noticeable bleaching, and we generally operate with the laser filtered to either $3 \%$ or $1 \%$. To compensate for the reduced intensity that results from the minimized opening of the confocal aperture, we increase the gain of the photomultiplier, and to compensate for the resultant increase in noise, we perform Kalman filtering during image collection so that each image is integrated over multiple scans (usually three).

For optimal resolution and image accuracy, data in the image plane should ideally be collected with a pixel size equal to one-half or less the theoretical lateral resolution imposed by the objective NA (Inoue, 1986; Gard, 1993). In confocal imaging systems, the lateral pixel dimension can be adjusted effortlessly using the microscope's computerized control software. (Physically, the angle at which the laser hits the sample is changed, so that the illuminated area is reduced. For example, with a $\times 60$ objective, one can achieve a final magnification equivalent to that achieved with a $\times 100$ objective using conventional light microscopy). But as noted above, the vertical resolution of confocal imaging systems is always less than the theoretical lateral resolution, and yet we would like to gather data such that the axial voxel dimension is equal to the lateral dimension. In our work we have generally sought to maximize the vertical resolution and accuracy.

In our experience, the vertical depths to which optical sectioning can be performed are variable and a function of the imaged material. For example, penetration depths of hundreds of microns $(\sim 250 \mu \mathrm{m})$ can be achieved with sandstone before significant image degradation is apparent. However, we observed penetration depths of only tens of microns $(\sim 50 \mu \mathrm{m})$ during our imaging of very fine fibrous insulations where the typical fiber dimension is only slightly larger than the excitation wavelength of the light source (see next section).

In comparison to the alternative porous media characterization methods discussed earlier, the confocal imaging technique has several advantages. First, confocal imaging currently offers the highest resolution of any of the 3D imaging methods available. Furthermore, confocal systems are relatively inexpensive and therefore more widely available. Sample preparation is simple, and the rapid data acquisition time and absence of facility time restrictions expedites analysis of multiple samples and/or sample sub-volumes. Finally, post-processing is relatively straightforward since image data are acquired on a voxelby-voxel basis, and because the data are intrinsically binary even in multiphase rocks. However, a significant limitation of LSCM compared to other direct imaging techniques such as MRI and synchrotron microtomography is the limit on the $z$-extent of the imaged volume due to absorption and scattering of the laser by the material overlying the optical plane.

\section{Application to porous media}

To illustrate some of the unique capabilities of confocal imaging, here we show several examples of its application. All of the data sets shown were collected with a Bio-Rad MRC-600 confocal imaging system fitted to a Nikon Diaphot, with a $568 \mathrm{~nm}$ excitor filter, YHS filter block, and with the opening of the confocal aperture at or very close to 


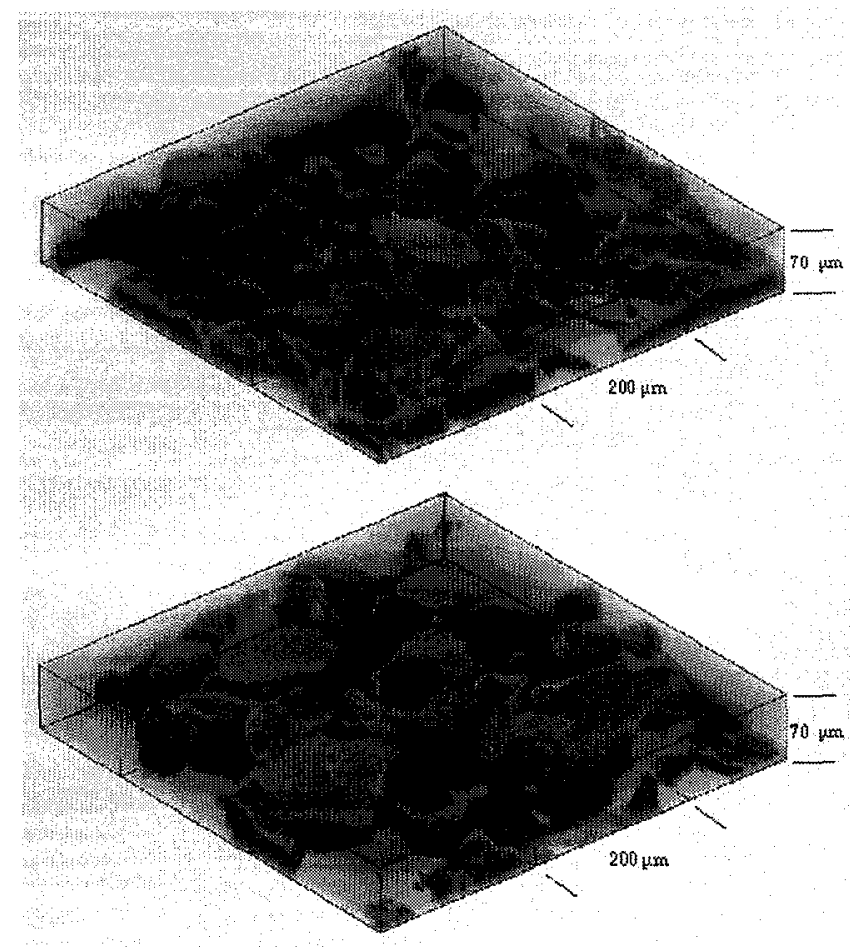

Fig. 3. 3D volume renderings of pore space in Berea sandstone. The pore phase is opaque, and solid grains are translucent. The images are $512 \times$ $512 \times 71$ voxels, with $(x \times y \times z)$ volumes of $0.526 \times 0.526 \times 0.071 \mathrm{~mm}^{3}$.
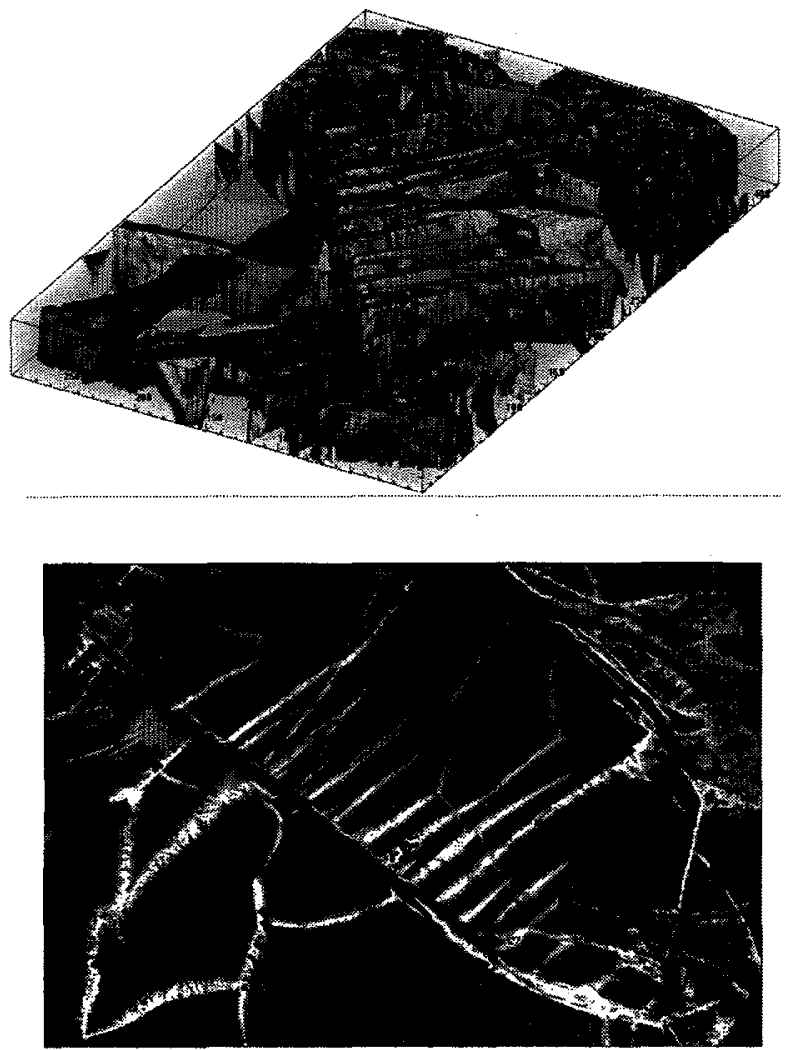

Fig. 4. (Top) 3D volume rendering of crack damage in a triaxially deformed rock (Spruce Pine dunite). The fine crack network in the upper right corner would not be resolvable using conventional LM. The image is $768 \times 512 \times 71$ voxels, with an image volume of $430 \times 287 \times 14.4 \mu^{3}$. (Bottom) A 2D image slice from the 3D data set. The relationship of the cracks trending NE-SW to the grain boundary (running diagonally NW$\mathrm{SE}$ ) is indisputable in the 3D image, but less so in the 2D slice. its minimal setting $(0.6 \mathrm{~mm})$. Collection times for the volumetric data arrays were in all cases $<1$ hour. The threedimensional reconstructions were performed using VoxelView 2.5 (Vital Images, Inc.) on a Silicon Graphics High Impact workstation.

Figure 3 shows two data sets acquired from a Berea sandstone sample with cubic voxels with dimension $1 \mu \mathrm{m}$. For clarity in visualizing the microgeometry of the pore space, the pore phase is shown as opaque, whereas the solid grains are translucent. The data illustrate clearly the fine structure and complicated topology and geometry of the pore space. Particularly conspicuous are the thin pore necks (throats) that connect the larger (nodal) pore bodies, and the generally irregular geometry of the larger pore bodies. As an example of the qualitative insights that can be derived consider that it is only with the addition of a third dimension that can we distinguish with certainty the nodal pore bodies from the channels connecting those nodal pores. A specific example is in the upper corner of the top image, where a prominent tubular pore connects two nodal pore bodies; this tubular pore would be indistinguishable from a spherical pore in any single two-dimensional image.

In Figure 4 we show a confocal volumetric image (top) from a low-porosity crystalline rock stressed under deviatoric all-compressive loading conditions in a triaxial deformation apparatus at very high confining pressure. The lateral voxel dimension is $0.56 \mu \mathrm{m}$, and the spacing between sections is $0.2 \mu \mathrm{m}$. With the addition of the third dimension it is clearly revealed that the micromechanical response is dominated by the growth of tensile cracks, with a remarkably fine crack spacing that is highly variable locally. The true crack geometry is difficult to infer from a single 2D image (bottom) because of the fine crack apertures and extremely high crack density. Moreover, using conventional LM, the sub-micron microcrack structure in the upper right would likely yield an undulatory texture suggestive of crystalline plasticity as opposed to brittle crack growth (e.g., Tullis and Yund, 1992).

In Figure 5 we demonstrate application to a porous engineering material that forms part of the Thermal Protection System (TPS) of hypersonic vehicles and spacecraft. Note that in these reconstructions, the solid phase is shown as opaque, whereas the pore phase in translucent. The insulation is made from a slurry of ceramic fibers that are pressed and then fired to yield a rigid material (Figure 1) with a bulk porosity of $>80 \%$ and a complex, anisotropic microstructure (Marschall and Milos, 1997). Central to the performance of TPS insulations is the maximization of their insulative properties with the minimization of their density, and quantitative characterization of the microstructure is thus critical for TPS modeling, development, and optimization. Because of the very fine microstructure (fiber diameters of a few microns with lengths of tens of microns), confocal microscopy is currently the only feasible technique for obtaining quantitative descriptions of the 3D microgeometry of both the solid and void phases. The confocal data (Figure 5) resolve clearly the complex 

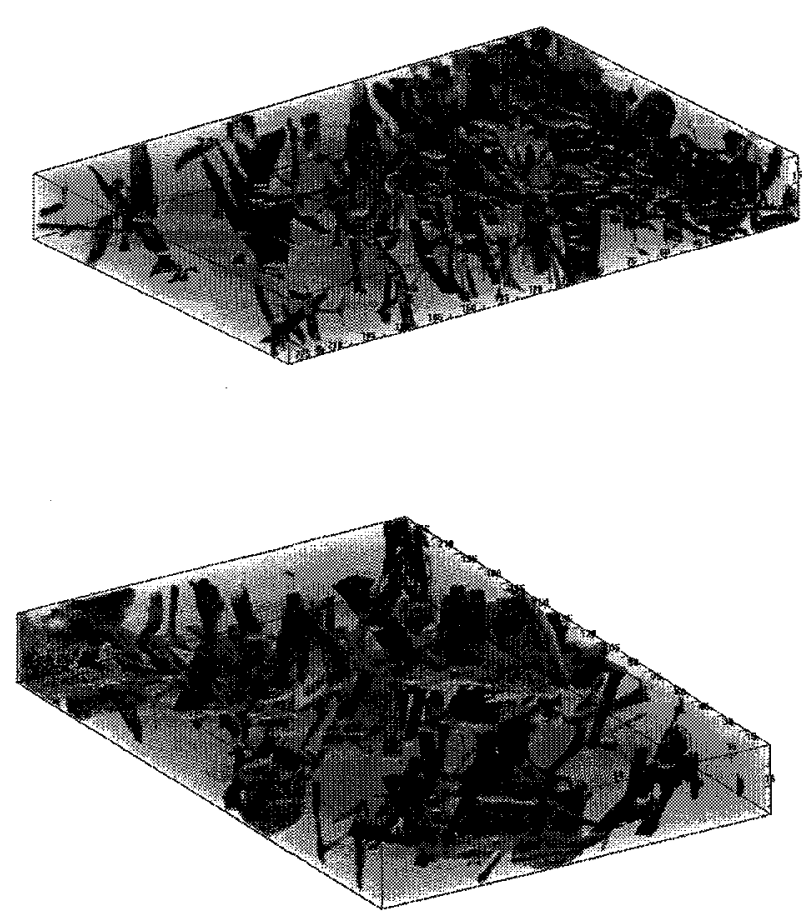

Fig. 5. 3D volume renderings of the solid phase in two rigid ceramic insulations. Each image is $768 \times 512 \times 85$ voxels, with cubic voxels with dimension $0.3 \mu \mathrm{m}\left(230 \times 154 \times 25.2 \mu \mathrm{m}^{3}\right)$. The two insulations are composed of the same three fiber constituents, but have been pressed to different final densities. The insulation shown at top has a bulk density of $0.35 \mathrm{~g} / \mathrm{cc}$ and bulk porosity of 0.86 , whereas that shown at bottom has a bulk density of $0.19 \mathrm{~g} / \mathrm{cc}$ and bulk porosity of 0.92 .

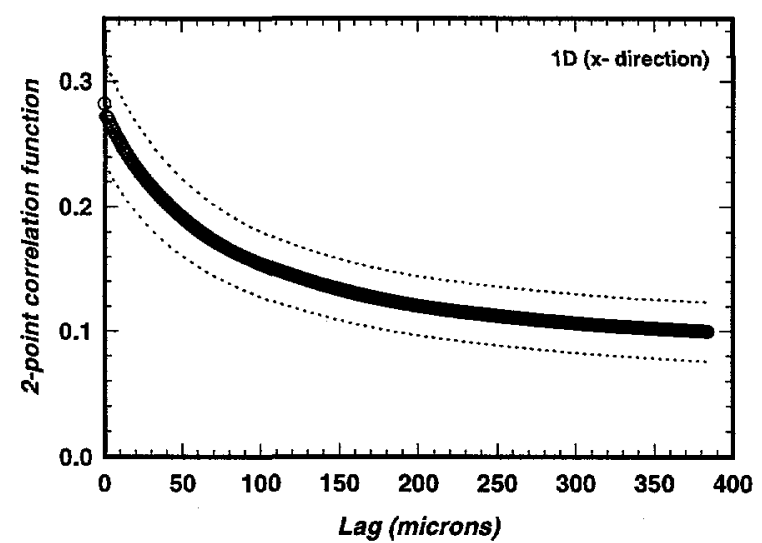

Fig. 6. Two-point correlation function $S_{2}$ calculated from 3D confocal image data for Berea sandstone. The 3D data set is $768 \times 512 \times 101$ voxels in size with cubic voxels of dimension $1 \mu \mathrm{m}$. The average porosity for the image volume (equal to the $y$-intercept) is $28 \%$, and the specific surface area (inversely proportional to the slope at the origin) is $23 \mathrm{~mm}^{2} / \mathrm{mm}^{3}$. The upper and lower curves represent one standard deviation. The point at which $S_{2}$ reaches an asymptotic values defines a characteristic length for the microstructure $(\sim 250 \mu \mathrm{m})$.

topology of the fibrous microstructure, and, ultimately, can be used to quantify the microgeometry (Fredrich and Marschall, 1999).

\section{Statistical microgeometric characterization}

Although the reconstructions provide important qualitative insights into the microstructure, an important application of the $3 \mathrm{D}$ image data is quantitative characterization of the pore space. Extracting statistical descriptions of the microgeometry from the volumetric image data requires rigorously segmenting the data on a voxel-by-voxel basis into solid or pore space. Quantitative analysis is complicated greatly by the finite voxel resolution that results in some voxels encompassing both solid and void space. This leads to a non-binary data distribution so that the fluorescence data are distributed across the available (8bit) range of $0-255$. This "partial voxel" problem is intrinsic to all imaging and tomographic techniques. A related difficulty caused by the finite voxel resolution is the blurring of edges.

Fredrich and Lindquist (1997) described the approaches that we have applied for segmenting 3D confocal data. A three-dimensional anisotropic diffusion algorithm that assumes Gaussian blurring is used to first "sharpen" the image data, and the data can then be segmented using either a uniform thresholding criteria, or, preferably, based upon a characteristic feature of the fluorescence histogram such as identification of a local minima. More recently, $O h$ and Lindquist (1998) have implemented a 3D kriging-based algorithm that relies on a local determination of the twopoint correlation function given a priori identification of a portion of the voxel population as void and solid phases. Our recent work with the latter approach has been particularly encouraging. All three methods are implemented in the $3 \mathrm{dma}$ code developed by Lindquist and co-workers (Lindquist et al., 1996; Lindquist and Venkatarangan, this volume) that we use to analyze our confocal image data.

Once the data have been segmented (i.e., binarized), firstorder descriptions of the microgeometry, that is, the porosity $\phi$ and specific surface area $S_{A}$, can be calculated readily using the $n$-point probability functions (e.g., Berryman, 1985). The one-point probability function $S_{1}$ is the probability that a voxel is located in the void phase. It is thus equal to the volume fraction of the void phase $(\phi)$, and can be determined by a straight voxel count. The two-point correlation function $S_{2}$ is the probability that any two points separated by a distance $r$ lie in the same phase:

$$
S_{2}(r)=\langle f(x) f(x+r)\rangle
$$

where the brackets denote volume averaging over the position vector $x$, and where the function $f$ is equal to 0 if $x$ lies in the solid phase and 1 if $x$ lies in the void phase. $S_{2}$ has the important attributes:

$$
\begin{gathered}
S_{2}(0)=S_{1}=\phi \\
\left.\frac{d}{d r} S_{2}(r)\right|_{r=0}=\frac{1}{4} S_{A}
\end{gathered}
$$



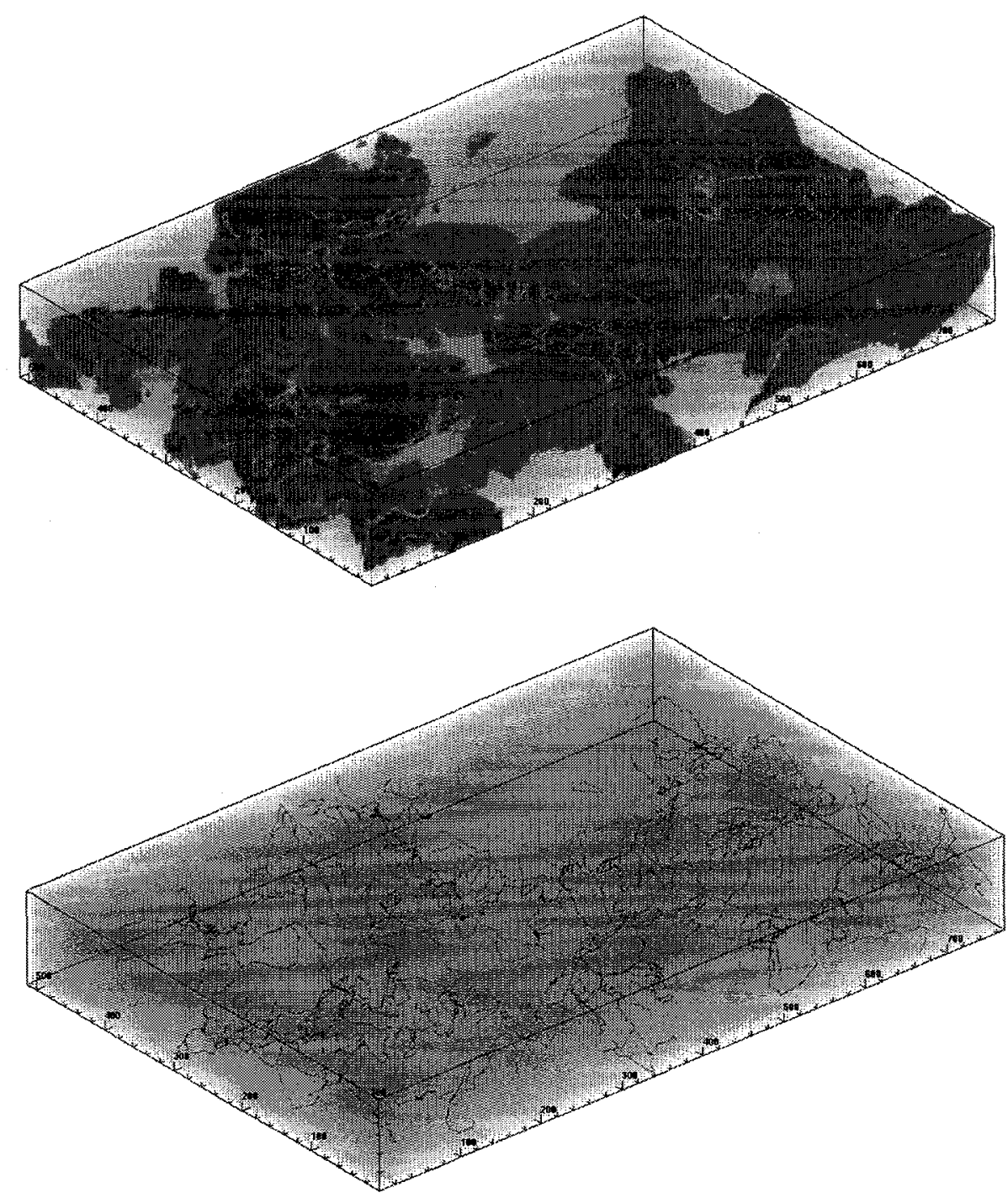

Fig. 7. (Top) 3D volume rendering of pore space in Berea sandstone. The data set is $768 \times 512 \times 101$ voxels in size, with cubic voxels of dimension $1 \mu \mathrm{m}$. The 2-point correlation function calculated for this volume is shown in Fig. 6. (Bottom) The corresponding medial axis calculated for the pore phase. The medial axis encodes the distance to the pore-solid interface, so that the geometry of the original structure is retained, and it consists of a series of "links" (or "paths") that connect and/or fork at "branch points" (or "branch clusters"). For example, consider a regular 2D cubic network consisting of nodes and bonds; in a medial axis representation, the nodes would correspond to branch clusters and the bonds to paths. In like manner, a three-dimensional geometrically complex (real) porous medium such as the sandstone considered here reduces to a topologically complex medial axis that consists of an irregular network of pore paths that interconnect at branch clusters.

$S_{2}$ can be calculated in 1D, 2D, or 3D (Figure 6), and variation in $S_{2}$ along the primary $x, y$, and $z$ axes can be used to test for isotropy, or conversely, to quantify anisotropy. Finally, the shape of the correlation function $S_{2}$ defines a characteristic length of the microstructure.

Developing efficient approaches and defining metrics to characterize the three-dimensional geometry and topology of porous media is challenging (e.g., Adler, 1992; Thovert et al., 1993). To derive higher order statistical metrics that characterize spatially distributed geometric aspects of the pore space, we have adopted the medial axis analysis (Lee et al. . 1994) as implemented computationally by Lindquist et al. (1996).

The medial axis of a volume-filling object can be thought of as the skeleton of the object running along its geometrical middle, and it retains both the topology and geometry of the original structure. The medial axis can be calculated for both the solid or pore phase, and it can be probed much more efficiently than the original object to obtain statistical descriptions of the three-dimensional microgeometry. In Figure 7 we show another 3D pore volume rendering for Berea sandstone, and the corresponding medial axis calculated for this pore volume.

Lindquist et al. (1996; this issue) discuss various statistical metrics that can be extracted using medial axis analysis, including grain and pore size distribution, coordination, pore path lengths, minimum throat size, etc. We are currently applying medial axis analysis to analyze systematically the microgeometry of the pore space as a function of diagenesis in natural Fontainebleau sandstone 


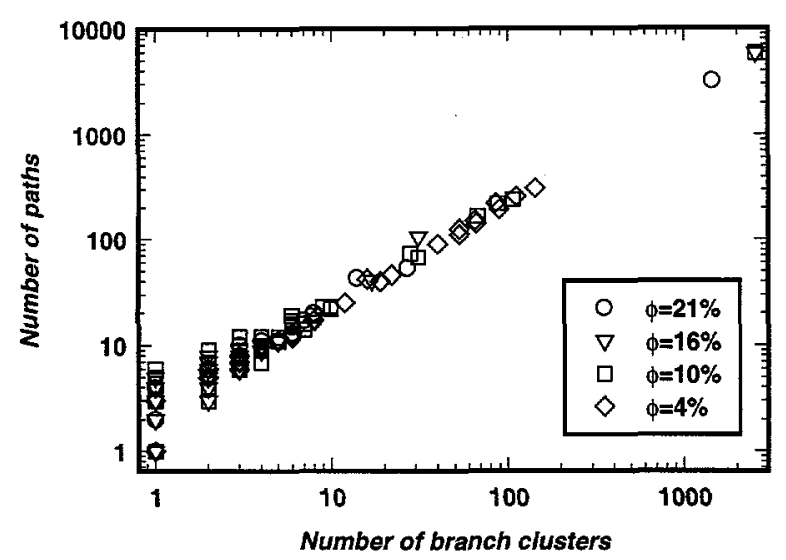

Fig. 8. Number of paths versus number of branch clusters (see Fig. 7 for definitions) for disconnected segments of the medial axis for four samples of a pure quartz sandstone (Fontainebleau) with bulk porosities of $21 \%$, $16 \%, 10 \%$, and $4 \%$ (Fredrich and Lindquist, 1997). The medial axis for the three higher porosity samples has a single segment that dwarfs the other segments and contains more than 3000 paths and $>1000$ branch clusters, and that corresponds to over $99 \%$ of the pore volume. However, the largest single segment of the medial axis for the lowest porosity sample represents only $21 \%$ of the total pore space, and contains only $\sim 500$ paths and $<200$ branch points. This sample lies below the transition from a power law relationship between porosity and permeability (Zhu et al., 1995), and is in the regime where Kozeny-Carman equivalent channel models over-predict bulk permeability by nearly two orders of magnitude (Fredrich et al., 1993).

and glass bead packs sintered to varying porosity.

As an example of our results, Fredrich and Lindquist (1997) applied medial axis analysis to 3D confocal image data for Fontainebleau sandstone, and showed quantitatively that the internal connectivity of the pore space undergoes drastic evolution with decreasing porosity. 3D image data were acquired for four samples of Fontainebleau sandstone with porosities of $21 \%, 16 \%$, $10 \%$, and $4 \%$, and following segmentation, medial axes were calculated for the pore phase for each of the image volumes. The medial axes for each image volume were then analyzed to determine the number of paths and number of branch clusters for each disconnected segment. They found that over the scale of the imaged volumes, over $99 \%$ of the pore space is internally connected for the three samples of Fontainebleau sandstone with porosities of $21 \%, 16 \%$, and $10 \%$. However, the largest segment of the medial axis for the sample with a porosity of $4 \%$ corresponds to only $\sim 22 \%$ of the total pore volume in the imaged domain (Figure 8). Further, this largest medial axis segment contains about an order of magnitude fewer branch clusters and pore paths than the largest medial axes segments in the three higher porosity samples.

As discussed by Zhu et al. (1995), the sample with $4 \%$ porosity is in the regime where the power law relationship (with an exponent $\sim 3$ ) between porosity and permeability is no longer obeyed, and instead permeability undergoes an accelerating decline with porosity reduction (Fredrich et al., 1995). Furthermore, Fredrich et al. (1993) showed that the Kozeny-Carman equivalent channel model (Walsh and Brace, 1984) over-predicts the bulk permeability of this sample by nearly two orders of magnitude, implying that the pore phase does not uniformly accommodate the bulk fluid flow. In turn, the medial axis analysis reveals quantitatively the decreased connectivity of the pore space, and suggests that the accelerated permeability reduction is due to the resultant increased tortuousity of the fluid flow paths.

\section{Summary}

Understanding the physics of microscale flow processes is central to many geoscience, chemical, materials, and thermal engineering applications. Recent developments in three-dimensional imaging offer an unprecedented opportunity to determine the microgeometry of porous media at high resolution. In this paper we have summarized the technical and practical considerations for applying laser scanning confocal microscopy to porous media, and demonstrated its application to various geologic and engineering materials. Although confocal microcopy has limited ability to penetrate solid materials, it is nevertheless the highest resolution technique currently available for three-dimensional imaging. Other benefits include the wide availability of the technology, and the relatively simple sample preparation requirements. The size of the volumetric data sets attainable using confocal microscopy are in any event of sufficient size ( $\sim 50$ million voxels) that they challenge significantly the current capabilities of highend workstations.

We believe that besides statistical characterization of the three-dimensional geometry of the microstructure, fundamental understanding of flow processes in complex porous media also requires study of the geometry of the flow itself. Although MRI has been applied successfully to image flow (local fluid velocity) in packed beds (Lebon et al., 1996a,b), the current resolution of MRI is typically not sufficient for imaging transport processes in complex geologic media.

Numerical simulation is the most promising technique at this time, and to this end, we have developed a process to automatically generate compact numerical meshes from 3D confocal image data. The principal issues regarding the tractable computation of transport phenomena in arbitrarily complex porous media lie in the complexity of the geometry and the retention of this structure in the analysis. Lattice Boltzmann methods have arisen as the most attractive approach for simulating flow in complex geometric domains due to the method's unique ability to treat simply and efficiently the multitude of discrete boundary conditions. O'Connor and Fredrich (this volume) discuss the application of Lattice Boltzmann methods for modeling heat and mass transport processes in these data sets. The simulations are used to predict macroscopic properties such as permeability, and volume visualization software can be used to study the three-dimensional flow geometry in the complex geometric domains characteristic of real porous media. Laboratory measurements of bulk flow properties are also performed for direct comparison 
with the numerically computed flow properties.

Ultimately, our aim is to derive new understanding of transport and flow in complex porous media by complimentary analyses of the 3D microgeometry, laboratory transport experiments, and pore-scale numerical flow simulations.

Acknowledgements. My work in this area over the last two years has benefited from productive and enjoyable collaborations with $\mathrm{W}$. Brent Lindquist (SUNY Stony Brook) and Ruaidhri M. O'Connor (Sandia). The work on ceramic insulations is being conducted in collaboration with Jochen Marschall (NASA Ames). I thank Christian David, David Olgaard, and Angel Rodriguez-Rey for their kind invitation to present this paper at their symposium. Comments by an anonymous reviewer and Teng-fong Wong improved the manuscript. Funding from the Engineering Sciences Research Foundation at Sandia (Laboratory Directed Research and Development Program) and the U.S. DOE Office of Basic Energy Sciences (Geosciences Program) is gratefully acknowledged. This work was performed at Sandia National Laboratories funded by the U.S. Department of Energy under Contract No. DE-AC04-AL85000. Sandia is a multiprogram laboratory operated by Sandia Corporation, a Lockheed Martin Company, for the United States Department of Energy.

\section{References}

Adler, P.M., Porous Media: Geometry and Transports, 544 pp., Butterworth-Heinemann Series in Chemical Engineering, Stoneham, Mass., 1992.

Auzerais, F.M., Dunsmuir, J., Ferreol, B.B., Martys, N., Olson, J., Ramakrishnan, T.S., Rothman, D.H., and Schwartz, L.M., Transport in sandstone: A study based on three dimensional microtomography, Geophys. Res. Lett., 23, 705-708, 1996.

Avnir, D., Farin, D., and Pfeifer, P., Surface irregularity of particulate materials: A fractal approach, J. Colloid Interface Sci., 103, 112-123, 1984.

Baldwin, C.A., Sederman, A.J., Matntlle, M.D., Alexander, P., and Gladden, L.F., Determination and characterization of the structure of a pore space from 3D volume images, $J$. Colloid Interface Sci., 181, 7992, 1996.

Bernabe, Y., Pore geometry and pressure dependence of the tranpsort properties in sandstones, Geophys., 56, 436-446, 1991

Berryman, J.G., Measurement of spatial correlation functions using image processing techniques, J. Appl. Phys., 57, 2374-2384, 1985.

Berryman, J.G. and Blair, S.C., Use of digital image analysis to estimate fluid permeability of porous materials: Application of two-point correlation functions, J. Appl. Phys. 60, 1930-1938, 1986.

Bourbie, T. and Zinszner, B., Hydraulic and acoustic properties as a function of porosity in Fontainebeau sandstone, J. Geophys. Res., 90, 11524-11532, 1985.

Brakenhoff, G.J., Blom, P., and Barends, P., Confocal scanning light microscopy with high aperture immersion lenses, $J$. Microsc., 117, 219$232,1979$.

Brakenhoff, G.J., van der Voort, H.T.M., van Spronsen, E.A., and Nanninga, N., Three-dimensional imaging in fluorescence by confocal scanning microscopy, J. Microsc., 153, 151-159, 1989.

Brown, G.O., Stone, M.L., and Gazin, J.E., Accuracy of gamma ray computerized topography in porous media, Water Res. Res., 29, 479486, 1993.

Brunauer, S., Emmett, P.H., and Teller, T., The adsorption of gases in multi-molecular layers, J. Am. Chem. Soc., 69, 309-316, 1938.

Cohen, M.H., and Mendleson, K.S., Nuclear magnetic relaxation and the internal geometry of sedimentary rocks, J. Appl. Phys., 53, 1127, 1982.

Coker, D. A., Torquato, S. and Dunsmuir, J. H., Morphology and physical properties of Fontainebleau sandstone via tomographic analysis, $J$. Geophys. Res., 101, 17497-17506, 1996.

David, C., Geometry of flow paths for fluid transport in rocks, J. Geophys. Res., 98, 12267-12278, 1993.
Doughty, D.A., and Tomutsa, L., Imaging pore structure and connectivity in high resolution NMR microscopy, Int. J. Rock Mech. \& Min. Sci., 34:3-4, 1997.

Doyen, P.M., Permeability, conductivity,and pore geometry of sandstone, J. Geophys. Res., 93, 7729-7740, 1988.

Dullien, F.A.L., Porous Media - Fluid Transport and Pore Structure, 2nd ed., Academic Press, New York, New York, 1992.

Fredrich, J.T., Greaves, K.H., and Martin, J.W., Pore geometry and transport properties of Fontainebleau sandstone, Int. J. Rock Mech. Min. Sci.., 30, 691-697, 1993.

Fredrich, J.T., Martin, J.W., and Owen, L.B., Application of X-ray computerized tomography for non-destructive measurement of physical properties of NTS tuff, Defense Nuclear Agency, DNA-TR-93-189, 121 pp., Alexandria, Virginia, 1994.

Fredrich, J.T., Menendez, B., and Wong, T.-F., Imaging the pore structure of geomaterials, Science, 268, 276-279, 1995.

Fredrich, J.T. and Lindquist, W.B., Statistical characterization of the threedimensional microgeometry of porous media and correlation with macroscopic transport properties, Int. J. Rock Mech. \& Min. Sci., 34(34), 1997.

Fredrich, J.T. and Marschall, J., Quantitative microstructural characterization of rigid fibrous insulations using laser scanning confocal micrsocopy, manuscript in preparation, 1999.

Gard, D.L., Confocal immunofluorescence microscopy of microtubules in amphibian oocytes and eggs, in Methods in Cell Biology, 38, 241-264, Aacademic Press, New York, NY, 1993.

Gratton, E. and vandeVen, J.J., Laser sources for confocal microscopy, in Handbook of Biological Confocal Microscopy, Pawley, J.B. (ed.), 53 67, Plenum Press, New York, NY, 1990

Hall, P.L, Mildner, D.F.R., Borst, R.L., Small-angle scattering studies of the pore space of shaly rocks, J. Geophys. Res., 91, 2183-2192, 1986.

Inoue, S, Video Microscopy, Plenum Press, New York, NY 1986.

Inoue, S., Foundations of confocal scanned imaging in light microscopy, in Handbook of Biological Confocal Microscopy, Pawley, J.B. (ed.), 1 14, Plenum Press, New York, NY, 1990.

Koplik, J., Lin, C., and Vermette, M., Conductivity and permeability from microgeometry, J. Appl. Phys., 56, 3127-3131, 1984.

Krohn, C.E., Sandstone fractal and Euclidean pore volume distributions, $J$ Geophys. Res., 93, 3286-3296, 1988.

Krohn, C.E. and Thompson, A.H., Fractal sandstone pores: Automated measurements using scacnning-electron-microscope images, Phys. Rev. $B, 33,6366-6374,1986$

Lebon, L., Oger, L., Leblond, J., Hulin, J.P. Martys, N.S., Schwartz, L.M., Pulsed gradient NMR measurements and numerical simulation of flow velocity distribution in sphere packings, Phys. Fluids, 6, 293-301, 1996.

Lebon, L., Leblond, J., Hulin, J.-P., Martys, N.S., and Schwartz, L.M., Pulsed field gradient NMR measurements of probability distribution of displacement under flow in sphere packings, Mag. Reson. Imag., 14, 989-991, 1996

Lee, T.-C., Kasyap, R. L. and Chu, C. -N. , Building skeleton models via 3-D medial surface/axis thinning algorithms, CVGIP: Graphical Models and Image Processing, 56, 462-478, 1994.

Lin, C., Pirie, G., and Trimmer, D.A., Low permeability rocks: Laboratory measurements and three-dimensional microstructural analysis, $J$. Geophys. Res., 91, 2173-2181, 1986.

Lindquist, W.B., Lee, S.-M., Coker, D.A., Jones, K.W., and Spanne, P., Medial axis analysis of void structure in three-dimensional tomographic images of porous media. J. Geophys. Res., 101, 8297-8310, 1996.

Lindquist, W.B., and Venkatarangan, A., Investigating $3 d$ geometry of porous media from high resolution images, Phys. Chem. Earth, (this issue), 1999.

Marschall, J. and Milos, F.S., Gas permeability of rigid fibrous refractory insulations, Proc. $32^{\text {nd }}$ Thermophysics Conf., Amer. Inst. Aero. and Astro., Reston, VA, 1997.

Mendelson, K.S., Nuclear magnetic resonance in sedimentary rocks: Effect of proton desorption rate, J. Appl. Phys., 53, 6465-6466, 1982.

Montoto, M., Martinez-Nistal, A., Rodriguez-Rey, A., Fernandez-Merayo, N., and Soriano, P., Mirofractography of granitic rocks under confocal scanning laser microscopy, J. Micros. 177, 138-149, 1995.

Myer, L. R., Kemeny, J.M., Zheng, Z., Suarez, R., Ewy, R.T., and Cook, N.G.W., Extensile cracking in porous rock under differential compressive stress, in Micromechanical Modelling of Quasi-Brittle Materials Behavior, V. C. Li (ed.), Appl. Mech. Rev., 45, 263-280, 1992. 
O'Connor, R.M. and Fredrich, J.T., Microscale flow modelling in geologic materials, Phys. Chem. Earth (this volume).

$\mathrm{Oh}, \mathrm{W}$. and Lindquist, W.B., Image thresholding by indicator kriging, IEEE Trans. Pattern Anal. Machine Intell., submitted, 1998.

Pawley, J.B., Handbook of Biological Confocal Microscopy, Plenum Press, New York, 1990.

Pittman, E.D. and Duschatko, R.W., Use of pore casts and scanning electron microscopy to study pore geometry, J. Sed. Petr., 40, 1153$1157,1970$.

Russ, J.C., Practical Stereology, 186 pp., Plenum Press, New York, New York, 1986.

Sahimi, M. Flow and Transport in Porous Media and Fractured Rock, 482 pp., VCH, Weinheim, FRG, 1995.

Spanne, P., Thovert, J.F., C.J. Jacquin, Lindquist, W.B., Jones, K.W., and Adler, P.M., Sychrotron computed microtomography of porous media: Topology and transports, Phys. Rev. Lett. 73, 2001-2004, 1994.

Spurr, A.R., A low viscosity epoxy resin embedding medium for electron microscopy, J. Ultrastructure Res., 26, 31-43, 1969.

Thompson, A.H., Fractals in rock physics, Ann. Rev. Earth Planet. Sci., 19, 237-262, 1991

Thovert, J.F., Salles, J., and Adler, P.M., Computerized characterization of the geometry of real porous media: their discretization, analysis and interpretation, J. Microsc., 170, 65-79, 1993.

Tullis, J. and Yund, R., The brittle-ductile transition in feldspar aggregates: An experimental study, in Fault Mechanics and Transport Properties of Rocks, B. Evans and T.-F. Wong (eds.), 89-117, Academic Press, San Diego, CA, 1992.

Underwood, E.E., Quantitative Stereology, 274 pp., Addison Wesley, Reading, Massachusetts, 1970.

Van Brakel, I., Modry, S., and Svata, M., Mercury porosimetry: State of the art, Powder Technol., 29, 1-12, 1981.

Walsh, J. B. and Brace, W. F., The effect of pressure on porosity and the transport properties of rock, J. Geophys. Res., 89, 9425-943, 1984.

Wellington, S.L., and Vinegar, J.J., X-ray computed tomography, J. Pet. Tech., 39, 885-898, 1987.

Wilson, T., Optical sectioning in confocal fluorescent microscopes, $J$. Microsc., 154, 143-156, 1989.

Wilson, T., The role of the pinhole in confocal imaging systems, in Handbook of Biological Confocal Microscopy, Pawley, J.B. (ed.), 113126, Plenum Press, New York, NY, 1990.

Wong, P.-Z., Howard, J., and Lin, J.-S., Surface roughening and the fractal nature of rocks, Phys. Rev. Lett., 57, 637-640, 1986.

Wong, T.-F., Geometric probability approach to the characterization and analysis of microcracking in rocks, Mech. Mat., 4, 261-276, 1985.

Wong, T.-F., Fredrich, J.T., and Gwanmesia, G.D., Crack aperture statistics and pore space fractal geometry of Westerly granite and Rutland quartzite: Implications for an elastic contact model of rock compressibility, J. Geophys. Res., 94, 10,267-10,278, 1989.

Yale, D.P., Recent advances in rock physics, Geophys., 50, 2480-2491, 1985.

Zinszner, B., Johnson, P.A., Rasolofosaon, P.N.J., Influence of change in physical state on elastic nonlinear response in rock: Significance of effective pressure and water saturation, J. Geophys. Res., 102, 8105$8120,1997$.

Zhu, W., David, C., and Wong, T.-F., Network modeling of permeability evolution during cementation and hot isostatic pressing, J. Geophys. Res., 100, 15451-15464, 1995. 